\title{
Approach for Localizing Scatterers in Urban Drone-to-Drone Propagation Environments
}

\author{
Dennis Becker, Uwe-Carsten Fiebig, Lukas Marcel Schalk \\ Institute of Communications and Navigation \\ German Aerospace Center (DLR) \\ Oberpfaffenhofen-Wessling, Germany \\ dennis.becker@dlr.de
}

\begin{abstract}
In the near future an increasing number of unmanned aerial vehicles (UAVs) are expected to be integrated into urban airspace. Direct Drone-to-Drone (D2D) communication is a promising approach for exchanging information in order to prevent mid-air collisions especially in dense urban areas. For a reliable and efficient communication the fundamental propagation mechanisms must be understood and specific channel models be developed. In previous work we identified the origin of some multipath components (MPCs) in first wideband channel measurements by applying a geometrical signal path simulation considering the outline of buildings and recorded flight tracks. But the performance of this approach depends on the degree of simulated details and can easily get computationally expensive in order to identify the origin of all measured MPCs. Therefore, in this work we enhance the identification by jointly estimating the delay and doppler frequency probability density functions (PDFs) for each scatterer and localize their origins by transforming the estimation into the 3D Cartesian domain and intersecting the results with known objects. We show the feasibility of this approach by investigating the parameter dependency on the results under simulated conditions and then compare the results when being applied on real measurement data. For estimating key parameters of the MPCs, we employ the Kalman enhanced super resolution tracking algorithm (KEST) algorithm.
\end{abstract}

Index Terms-Unmanned Aerial Vehicle, Air-to-Air, Propagation, Drone-to-Drone Communication, Scatterer Localization

\section{INTRODUCTION}

The number of small sized UAVs is expected to increase significantly in the near future. Therefore, in order to integrate UAVs safely into urban air space, high reliable collision avoidance systems are mandatory. We see direct Drone-toDrone (D2D) communication as a promising approach for exchanging important information reliably with very low latency. In order to properly design such a D2D communication system, an accurate wideband channel model for the D2D link, specifically developed for the urban environment, is needed. We further aim to develop a geometry-based stochastic channel model, which includes geometrical relations as well as channel statistics and allows to model non-stationary propagation effects. To model the real world propagation behavior, we have conducted a channel propagation measurement campaign with two small sized UAVs flying in an urban environment at low heights and close to buildings. In previous work, we have proposed an experimental setup for D2D wideband channel measurements between small sized UAVs that overcomes limitations on the measurement equipment in terms of size, weight and power consumption [1] and have presented first findings on identifying multipath component sources for one flight scenario by geometrical signal path simulation [2]. But the geometrical signal path simulation lacks on the necessity of detailed information about the environment, which makes simulation relative complex and the identification of MPC sources impossible, if the related real world objects are unknown or not all underlying phyiscal effects are modeled. Therefore, in this work we localize the scatterer sources directly by generating the probability density function (PDF) of a joint delay and doppler frequency estimation for each scatterer and transforming it into the three dimensional Cartesian domain. Then we intersect the location estimates with the knowledge of the 3D geometry of known objects, which can be obtained by land surveying offices or from different online sources. The estimation is based on the fact that we can define points of constant delay lying on an ellipsoid with the transmit and receive antenna locations as focal points and points of constant Doppler frequencies lying on cones centered at the antenna locations. This approach has already been applied in different two dimensional scenarios [3], [4]. We extend this approach to the three dimensional domain and first evaluate the parameter dependency under simulated conditions with known MPCs by our signal path simulation and then show its feasibility on real measurement data. In order to estimate and track the multipath parameters such as delay, phase and lifetime, we employ the KEST [5] on the measurements.

\section{SCATtERER LocAlizATION ApProACH}

The algorithm for localizing a multipath component (MPC) $m p c_{i}$ from our measurements is based on their estimated signal propagation delay $\tau_{i}(t)$ and Doppler frequency $\nu_{i}(t)$. In theory in the $3 \mathrm{D}$ domain, for single-bounce reflections, the possible MPC source locations as the reflection points that lead to the same delay, lie on the surface of an ellipsoid defined by the delay $\tau_{i}(t)$ and the position $\mathrm{P}_{r}(t)$ and $\mathrm{P}_{t}(t)$ of the receiver $\mathrm{Rx}$ and the transmitter $\mathrm{Tx}$ as foci points of the ellipsoid. Furthermore, the reflection points, leading to the same Doppler frequency, are located on the surface of a cone defined by $\nu_{i}(t)$, which is fixed on the position of $\mathrm{P}_{r}(t)$. In principle, the shape of the ellipsoid is only dependent on the delay an the actual positions of receiver and transmitter, 
but the shape of the Doppler cone is also influenced by the movement, meaning direction and velocity, of both in the 3D domain. The intersection of the two defined surfaces then contains the true location plus the ambiguities. For point scatterers the true reflection locations do not change, when the receiver or transmitter is moving, whereas location of the ambiguities change. Therefore we make use of the idea to calculate the PDF of the MPC positions and average them for the time the MPC is visible like described in [3]. By averaging the PDFs, the ambiguities for point scatterer average out and therefore enhances the localization. If the true reflection location moves on a surface, then averaging might not enhance the localization, but the representation as PDF nevertheless helps to identify the most probable region of the true MPC location. Similar to [3], we represent the parameters $\tau$ and $\nu$ jointly as a bivariate Gaussian distribution

$$
p\left(\tau, \nu, \mu_{\tau}, \mu_{\nu}, \sigma_{\tau}, \sigma_{\nu}\right)=\frac{1}{2 \pi \sigma_{\tau} \sigma_{\nu}} e^{-\left(\frac{\left(\tau-\mu_{\tau}\right)^{2}}{2 \sigma_{\tau}^{2}}+\frac{\left(\nu-\mu_{\nu}\right)^{2}}{2 \sigma_{\nu}^{2}}\right)}
$$

by assuming that the parameters are independent to each other. The PDF is transformed into Cartesian domain by connecting the parameters $\tau$ and $\nu$ to it. The delay in the 3D Cartesian domain is calculated by the traveled distance

$$
d=\left\|\mathrm{P}_{s}-\mathrm{P}_{t}\right\|+\left\|\mathrm{P}_{r}-\mathrm{P}_{s}\right\|
$$

from transmitter position $\mathrm{P}_{t}$ to the reflection point $\mathrm{P}_{s}$ and the receiver position $\mathrm{P}_{r}$ with assuming speed of light $c$ as

$$
\begin{aligned}
\tau(x, y, z)= & \frac{d}{c} \\
= & \frac{1}{c}\left(\left\|\mathrm{P}_{s}-\mathrm{P}_{t}\right\|+\left\|\mathrm{P}_{r}-\mathrm{P}_{s}\right\|\right) \\
= & \frac{1}{c}\left(\sqrt{\left(\mathrm{x}_{s}-\mathrm{x}_{t}\right)^{2}+\left(\mathrm{y}_{s}-\mathrm{y}_{t}\right)^{2}+\left(\mathrm{z}_{s}-\mathrm{z}_{t}\right)^{2}}\right. \\
& \left.+\sqrt{\left(\mathrm{x}_{r}-\mathrm{x}_{s}\right)^{2}+\left(\mathrm{y}_{r}-\mathrm{y}_{s}\right)^{2}+\left(\mathrm{z}_{r}-\mathrm{z}_{s}\right)^{2}}\right)
\end{aligned}
$$

with all parameters variant on time t. The Doppler frequency can be expressed as

$$
\begin{aligned}
\nu & =-\frac{\delta}{\delta t} \tau f_{c} \\
& =-\frac{\delta}{\delta t} d \frac{f_{c}}{c}
\end{aligned}
$$

and reformulated in dependency of the velocity vectors of transmitter and receiver with

$$
\begin{aligned}
& \mathrm{P}_{t}(t)=\mathrm{P}_{t_{0}}+\mathrm{Vel}_{t} \cdot t \\
& \mathrm{P}_{r}(t)=\mathrm{P}_{r_{0}}+\mathrm{Vel}_{r} \cdot t
\end{aligned}
$$

and applying the time derivative to the distance $\mathrm{d}$ to

$$
\begin{aligned}
\nu= & -\frac{f_{c}}{c}\left(-\operatorname{Vel}_{t} \cdot \frac{1}{2\left\|\mathrm{P}_{s}-\mathrm{P}_{t}\right\|} \cdot 2\left(\mathrm{P}_{s}-\mathrm{P}_{t}\right)\right. \\
& \left.+\mathrm{Vel}_{r} \cdot \frac{1}{2\left\|\mathrm{P}_{r}-\mathrm{P}_{s}\right\|} \cdot 2\left(\mathrm{P}_{r}-\mathrm{P}_{s}\right)\right) \\
= & -\frac{f_{c}}{c}\left(-\mathrm{Vel}_{t} \frac{\left(\mathrm{P}_{s}-\mathrm{P}_{t}\right)}{\left\|\mathrm{P}_{s}-\mathrm{P}_{t}\right\|}+\operatorname{Vel}_{r} \frac{\left(\mathrm{P}_{r}-\mathrm{P}_{s}\right)}{\left\|\mathrm{P}_{r}-\mathrm{P}_{s}\right\|}\right)
\end{aligned}
$$

Note, that for the correct transformation of the PDF from parameter domain into Cartesian domain, a normalization factor is necessary in order to represent the extend of each infinitesimal point from the one domain in the other domain. But as we only have 2D PDF in the parameter domain, we lack of information to correctly transform each point into the $3 \mathrm{D}$ Cartesian domain and the resulting PDF might be distorted. But our results show, that the transformed PDF without normalization seems feasible for the localization approach. By calculating the PDF of the MPC location at a certain measurement time or the averaged PDF over the full life time of the scatterer and intersecting it with a 3D layout of the measurement environment, we can then estimate the most probable locations. If only a $2 \mathrm{D}$ layout of the environment is available by satellite images, then the PDF can be plotted by its layers of different heights on top of the satellite image in order to achieve height-dependent intersections. In order to calculate the MPC position estimates from our measurement data, we apply the KEST algorithm on the measured channel impulse response in order to get information about the mean values and variances for delay and phase for each detected MPC. The Doppler frequency is calculated from the change of the estimated phase from measurements with

$$
\nu=-\frac{\delta}{\delta t} \frac{\Phi}{2 \pi}
$$

\section{RESUlTS AND Discussion}

In order to verify the localization approach and analyze its performance for a urban D2D propagation in a 3D layout, we apply it on measurement data from our wideband channel measurement campaign described in [1], [2] as well as on simulated artificial scenarios. In [2] we already used an modeled environment for a ray-tracing simulation in order to identify most of the visible MPCs in the measurement data by comparing the calculated channel impulse response with the measured one. The already identified MPCs by raytracing for one flight scenario will help to verify the results. Therefore we will apply the scatterer localization approach first on artificial flight scenarios in our simulation and then compare these results to the real measurement data. Fig. 1 shows the geometrical environment and the drones flights tracks for one measured flight scenario, which is modeled for simulation with the surrounding buildings in a local Cartesian coordinate system.

\section{A. Analysis with Artificial Scenario}

For the artificial flight scenario, we model the measured scenario in our simulation. UAV TX is hovering on a certain point at $15 \mathrm{~m}$ height, whereas UAV RX flies at constant velocity of $0.5 \mathrm{~m} / \mathrm{s}$ towards UAV TX for about 20 seconds at same height. First we verify the location approach by investigating the results for the univariate Gaussian distributions for the delay and the Doppler frequency independent from each other. Fig. 2 shows the estimated probable locations for a scatterer, resulting from the surface of building B 103 for a certain instant of time, when considering only the delay information. The 


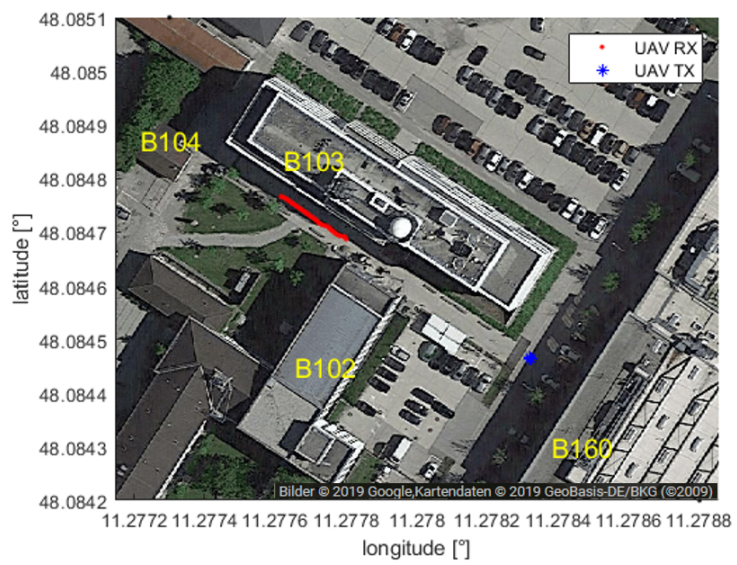

Fig. 1. Satellite image of environment and flight tracks of the measured flight scenario.

higher probable locations are indicated in red and the lower probable locations down to 10 percent of the maximum value are indicated in blue. The results clearly show the estimated delay ellipsoid, that is correctly located in the 3D Cartesian domain and the drone positions are in the foci points of it. Furthermore, fig. 3 shows the results, when considering only the Doppler frequency information. Thereby the picture is cut at the boundaries of the simulated environment. The picture shows the estimated Doppler frequency cone, that is fixed on the position of the receiving drone UAV RX and opens towards the simulated scatterer source at building B103. Fig. 4 shows the distribution of the joint delay and Doppler frequency estimation, which results in the intersection between the Delay ellipsoid and Doppler frequency cone. When averaging the joint distributions over all time instants, we can see in fig. 5 that the ambiguities decrease and the location estimation of the simulated scatterer becomes feasible, when intersecting the PDF with the environment. Note, that the scatterer is moving for the simulated 20 seconds, but its position change is small enough in order not being averaged out. As a result, the probable source locations are in a much more dense space and only a certain area on the surface of building B103 is the highest probable the source of the observed scatterer.

Furthermore, we simulate the single bounce reflections at the surfaces of the surrounding buildings B103, B104 and B160 plus two in [2] identified reflections resulting from stealbeams B160 SB1 and B160 SB2 on top of building B160 and the ground wave reflection GREF as shown in [2]. When applying the localization approach with averaging for the whole flight for all simulated MPCs and intersect it with known objects as illustrated in fig. 6, we can see that the localization approach is able to identify the true locations.

\section{B. Analysis with Measurement Data}

The flight tracks of the measured scenario, illustrated in fig. 1, only slightly differ from ones of the artificial scenario . Therefore, the results can be compared to each other. For estimating the delay and Doppler frequency for each MPCs,



Fig. 2. Delay ellipsoid for a scatterer at surface of building B103.

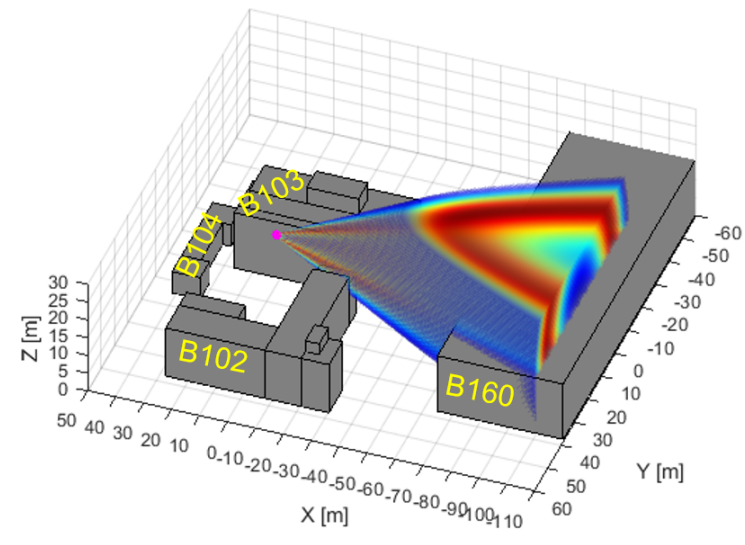

Fig. 3. Doppler frequency cone for a scatterer at surface of building B103, which is cut at certain boundaries for better presentation. The higher probable locations are indicated in red and the lower probable locations down to 10 percent of the maximum value are indicated in blue.

we apply the KEST algorithm on the measurement data for the given flight track. Fig. 7 plots the estimated delays and fig. 8 the Doppler frequencies for the most dominant MPC with indicated median values and the 75 th as well as the 25 th percentile. For the given measurement data, the scatterers resulting from the surfaces of building B104 and B103 could not be detected and also for the detected scatterers, KEST was not able to track them constantly for the whole time, leading to gaps in the delay information and bigger distortions in the phase information. Nevertheless, we are able to retrieve the Doppler frequencies for each scatterer and they are close to the expected values from simulation despite relatively high variances.

When applying the localization approach for all estimated MPCs and intersect it with known objects as illustrated in fig. 9, we can see that the uncertainty in the location estimation is much higher than in the simulated scenario. This results from the relative high Doppler frequency variances, leading to wider angles and thicker Doppler frequency cones. The 


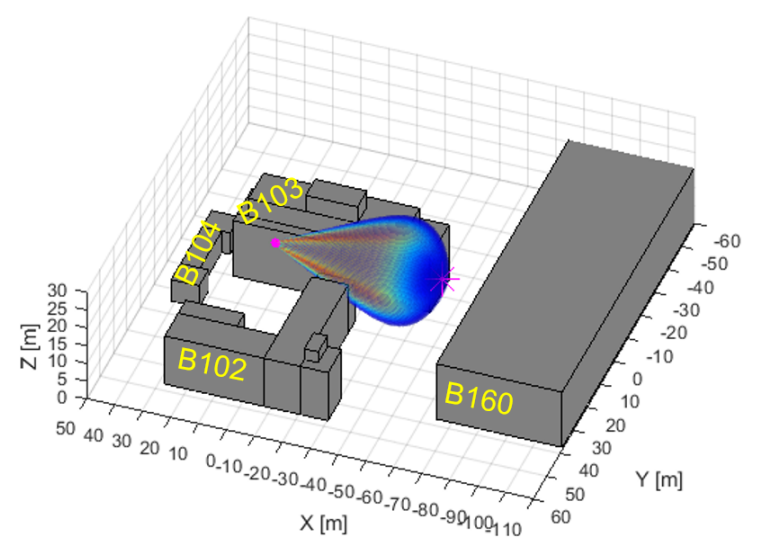

Fig. 4. Estimated location for a scatterer at surface of building B103 for one measurement snapshot.

scatterer location estimate for MPC B160 DREF is shown by the part at position $(x=-70, y=0)$. Whereas the other estimates at least match their true positions by their delay ellipse intersected with the objects, the results from B160 DREF do not intersect at all with any surface or object in the environment. This is due to double bounce reflection, resulting in a longer delay and changed Doppler frequency that leads to a non existent virtual scatterer location. Fig. 10 shows the results, when artificially decreasing the estimated variance of the Doppler frequency information. The location estimation improves, but when looking in details on the probability values, we can see that the highest probable locations for each MPC start to split up into two different locations among the delay ellipse. This results from the estimation error on the mean Doppler frequency values. Nevertheless, all estimated scatterer resulting from single bounce reflections can be identified when considering the underlying environment.

\section{CONCLUSION AND OUTLOOK}

In this paper we proposed an approach for localizing scatters for D2D propagation environments with 3D layout as in urban scenarios. The approach is based on a joint Doppler frequency and delay estimation by transforming the estimated PDF into the 3D Cartesian domain and intersecting the results with known objects. Artifical scenarios by simulation show, that the approach is feasible, but when applied on measurment data, its performance heavily depends on the parameter estimation for the delay and phase values of each scatterer. In our case the velocities for the drones in our measurement had to be relatively low leading to low Doppler frequencies that are hard to estimate. Nevertheless, the source of scatterer resulting from single bounce reflections could be identified. Further on we will investigate the parameter estimation in order to improve the localization approach for all other scenarios in our measurement data.

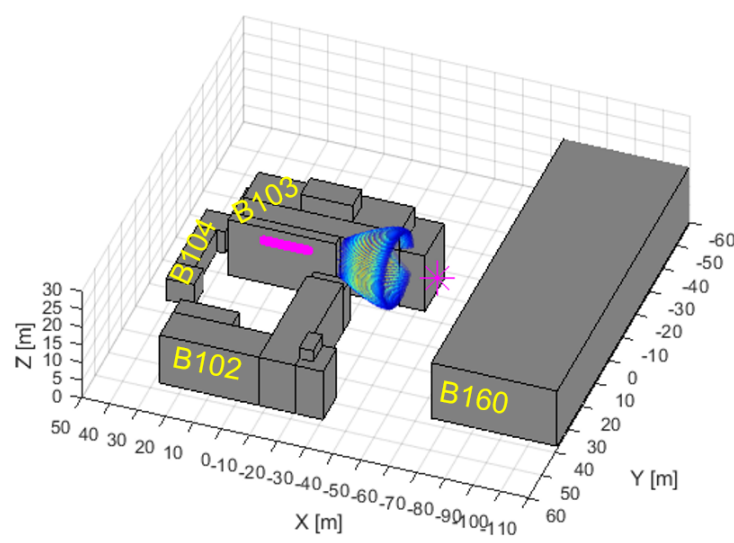

(a) 3D View

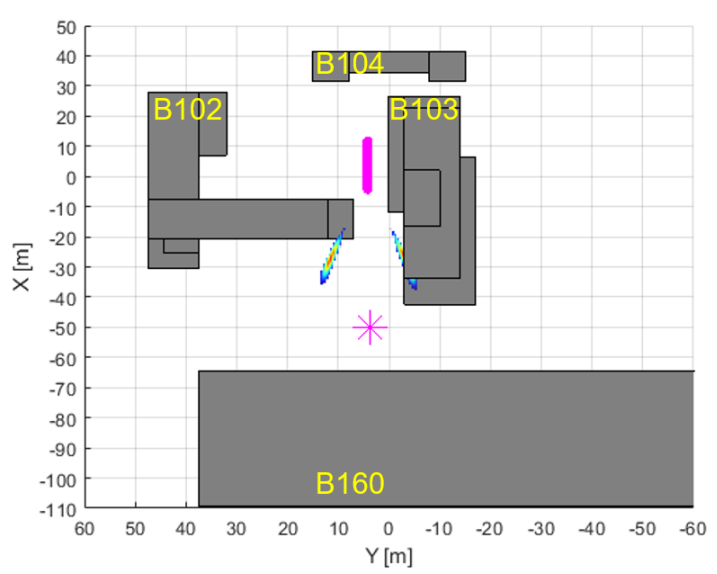

(b) 2D View at $15 \mathrm{~m}$ height

Fig. 5. Estimated location for scatterer at surface of building B103 with averaging over full flight.

\section{REFERENCES}

[1] D. Becker and L. Schalk, "Enabling Air-to-Air Wideband Channel Measurements between Small Unmanned Aerial Vehicles with Optical Fibers," 2019 IEEE/AIAA 38th Digital Avionics Systems Conference (DASC), San Diego, CA, USA, 2019, pp. 1-7

[2] D. Becker, U. Fiebig and L. Schalk, "Wideband Channel Measurements and First Findings for Low Altitude Drone-to-Drone Links in an Urban Scenario," 2020 14th European Conference on Antennas and Propagation (EuCAP), Copenhagen, Denmark, 2020, pp. 1-5

[3] N. Schneckenburger et al., "Reflector localization for geometrical modeling the air-ground channel," IEEE Transactions on Vehicular Technology, pp. 1-1, 2018

[4] Unterhuber, Paul and Walter, Michael and Schneckenburger, Nicolas and Kürner, Thomas, "Joint Delay and Doppler Frequency Estimation for Scatterer Localization in Railway Environments", 13th European Conference on Antennas and Propagation, EuCAP 2019

[5] T. Jost, W. Wang, U. C. Fiebig, and F. Perez-Fontan, "Detection and Tracking of Mobile Propagation Channel Paths," IEEE Transactions on Antennas and Propagation, vol. 60, no. 10, pp. 4875-4883, Oct 2012 


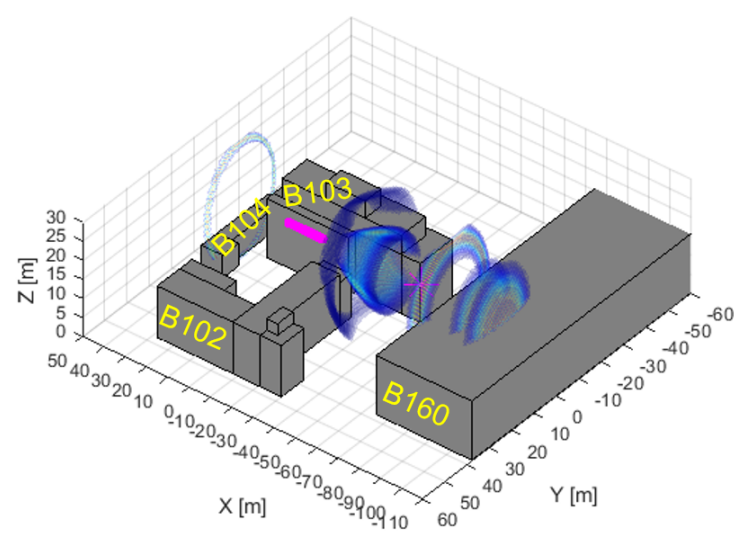

(a) 3D View



(b) 2D View

Fig. 6. Estimated location for all simulated scatterers with averaging over full flight. The higher probable locations are indicated in red and the lower probable locations down to 10 percent of the maximum value are indicated in blue.



Fig. 7. Estimated delays for detected scatterer by KEST algorithm.



Fig. 8. Estimated Doppler frequencies for detected scatterer by KEST algorithm with indicated mean values in red.

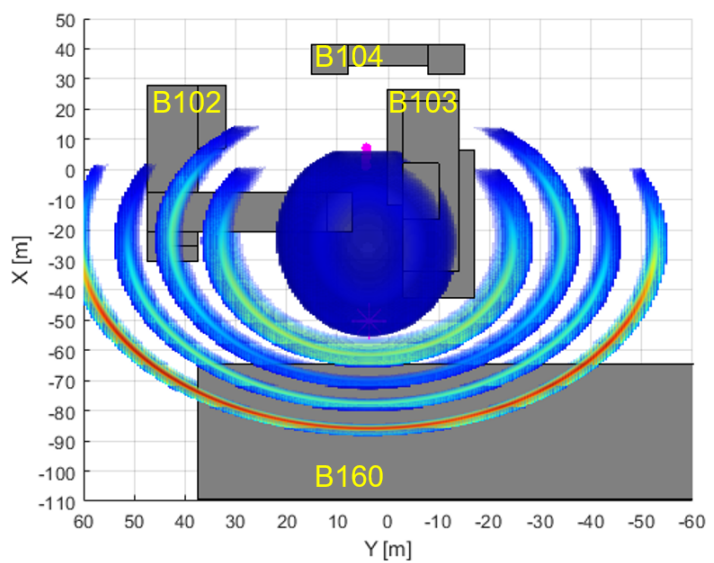

Fig. 9. Estimated location for all detected scatterers from measurement data.

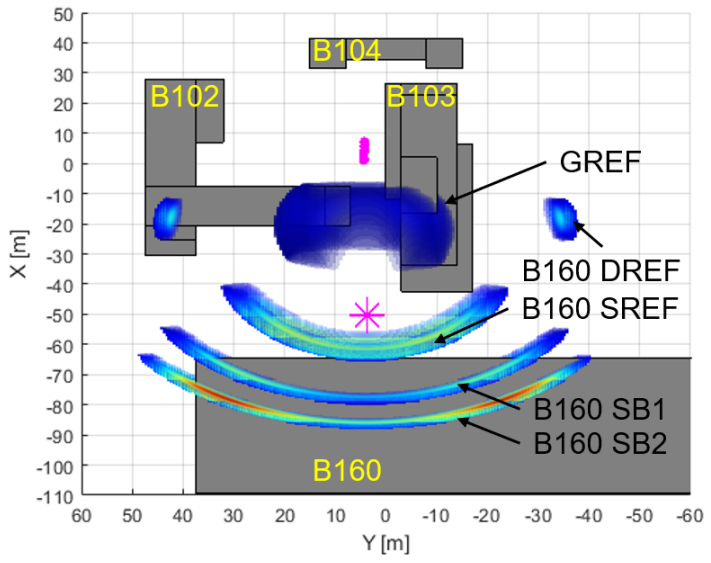

Fig. 10. Estimated location for all detected scatterers from measurement data with artificially decreased Doppler frequency variances. 